\title{
Estado nutricional antropométrico e comorbidades associadas à síndrome metabólica em mulheres submetidas à cirurgia bariátrica
}

\author{
Anthropometric nutritional status and comorbidities associated with metabolic \\ syndrome in women submitted to bariatric surgery
}

Julia de Castro Querido', Laís Rodrigues de Aguiar¹, Marina Pereira Justino', Sheilla de Oliveira Faria', Roberta Ribeiro Silva ${ }^{1}$, Cristiane da Silva Marciano Grasselli ${ }^{1} \bowtie$

${ }^{1}$ Faculdade de Nutrição, Universidade Federal de Alfenas. Alfenas, MG.

\section{RESUMO}

Objetivos: Avaliar o impacto da cirurgia bariátrica sobre o estado nutricional antropométrico e comorbidades associadas à síndrome metabólica e verificar a adequação do consumo de micronutrientes em mulheres submetidas ao procedimento.

Métodos: Estudo observacional, transversal, no qual foram avaliadas mulheres submetidas à cirurgia bariátrica. O consumo alimentar foi avaliado por meio de registro alimentar aplicado em triplicata. O estado nutricional antropométrico (peso, altura, índice de massa corporal e circunferência da cintura) foi aferido no período pós-cirúrgico. Dados sobre antropometria antes da cirurgia e presença de comorbidades (hipertensão arterial, diabetes mellitus, dislipidemia, doenças cardiovasculares e outras doenças) foram obtidos por questionário estruturado, padronizado e pré-codificado. Para avaliação dos resultados, as mulheres foram estratificadas em dois grupos considerando o tempo transcorrido após a cirurgia no momento da avaliação: G1 com tempo pós-cirúrgico até 36 meses e G2 com tempo pós-cirúrgico maior do que 36 meses. Resultados: Foram estudadas 14 mulheres, com média de idade de 34,6 14 anos, com tempo de realização da cirurgia compreendido entre 11 a 84 meses, sendo oito alocadas em G1 e seis em G2. Na avaliação após a cirurgia bariátrica observou-se redução do peso (p<0.01) e do índice de massa corporal $(\mathrm{p}<0.01)$ em ambos os grupos. Na avaliação pós cirúrgica, foram classificadas como obesas pelo índice de massa corporal $25,0 \%$ das mulheres do G1 (tempo de cirurgia até 36 meses) e 33,3\% das mulheres do G2 (tempo de cirurgia após 36 meses). Verificou-se diminuição das comorbidades associadas à síndrome metabólica entre as avaliações pré e pós cirúrgicas. Foram detectadas deficiências no consumo relatado de micronutrientes após a cirurgia, destacando-se cálcio, ferro e vitamina B12.

Conclusões: Os resultados apontam para a eficácia da cirurgia bariátrica no tratamento da obesidade e controle das comorbidades associadas à síndrome metabólica nesta amostra de mulheres. A presença de inadequações no consumo de nutrientes assinala a necessidade do cuidado nutricional no pós operatório da cirurgia bariátrica.

DESCRITORES: síndrome x metabólica; cirurgia bariátrica; obesidade; estado nutricional; comorbidades.

\section{ABSTRACT}

Aims: To assess the impact of bariatric surgery on anthropometric nutritional status and comorbidities associated with metabolic syndrome and to verify the adequacy of micronutrient intake of women undergoing this surgical procedure.

Methods: An observational cross-sectional study was performed to evaluate women undergoing bariatric surgery. Dietary intake was assessed using a food record in triplicate. The anthropometric nutritional status (weight, height, body mass index, and waist circumference) was measured in the postoperative period. Anthropometric data before surgery and presence of comorbidities (hypertension, diabetes mellitus, dyslipidemia, cardiovascular diseases, among others) were obtained by a structured, standardized, pre-coded questionnaire. To evaluate the results, women were stratified into two groups considering the time after surgery: less than or equal to 36 months (G1) and greater than 36 months (G2).

Results: Fourteen women with a mean age of $34.6 \pm 14$ years and postoperative time between 11 and 84 months were assessed; eight of whom were allocated to G1 and six to G2. Decreases in weight $(p<0.01)$ and body mass index $(p<0.01)$ were observed in both groups after bariatric surgery. Twenty-five percent of G1 women (up to 36 months after surgery) and 33\% of G2 women (more than 36 months after surgery) were considered to be obese. There were a lower number of comorbidities associated with metabolic syndrome when preoperative and postoperative assessments were compared. Micronutrient deficiencies (especially of calcium, iron, and vitamin B12) were detected after surgery.

Conclusions: Bariatric surgery was shown to be an effective method for treating obesity and controlling comorbidities associated with metabolic syndrome. Inadequate nutrient intake, however, indicates a greater need of postoperative nutritional care in patients undergoing bariatric surgery. KEY WORDS: metabolic syndrome X; bariatric surgery; obesity; nutritional status; comorbidity. 
Abreviaturas: SM, síndrome metabólica; DM 2, diabetes mellitus tipo 2; HDL-c, colesterol ligado à lipoproteína de alta densidade; IMC, índice de massa corporal; CC, circunferência da cintura; DRIs, Dietary Reference Intakes.

\section{INTRODUÇÃO}

A síndrome metabólica (SM) é atualmente definida como um agrupamento de anormalidades metabólicas que incluem obesidade central, hipertensão, resistência à insulina, hiperinsulinemia, diabetes mellitus tipo 2 (DM 2), diminuição do colesterol ligado à lipoproteína de alta densidade (HDL-c) e triglicérides séricos elevados [1].

Dentre os fatores que são mais comumente atribuídos ao desenvolvimento da SM estão a obesidade e resistência à insulina. A obesidade é considerada uma doença complexa, de múltiplas etiologias, constituindo um problema de saúde pública. Segundo a Organização Mundial da Saúde, em 2014 mais de 600 milhões (aproximadamente 13\%) de adultos no mundo estavam obesos. No Brasil, de acordo com pesquisa do Ministério da Saúde, 82 milhões de pessoas maiores de 18 anos estão acima do peso, ou seja, pouco mais da metade $(56,9 \%)$ da população do País [2-5].

Um tratamento ideal para obesidade levaria não só à perda de peso, mas também ao alívio das comorbidades associadas [6]. A cirurgia bariátrica é considerada uma ferramenta eficaz para o tratamento e controle da obesidade mórbida e também vem demonstrando efetividade para o controle da SM [2,7]. Isto porque a perda e a manutenção do peso a longo prazo são alvos primários para reverter beneficamente todos os componentes dessa síndrome [8]. Além disso, cada vez mais observa-se que os benefícios da cirurgia bariátrica, em particular nas comorbidades, são devidos não só à restrição calórica forçada, mas também a mecanismos neuro-hormonais mais sofisticados. Estudos têm documentado que as taxas de resolução de SM foram altas após a cirurgia bariátrica, variando de $66 \%$ a $84 \%[9,10]$.

A cirurgia bariátrica é indicada para pacientes cujo índice de massa corporal (IMC) seja igual ou superior a $40 \mathrm{~kg} / \mathrm{m}^{2}$ ou igual ou superior a $35 \mathrm{~kg} / \mathrm{m}^{2}$ com pelo menos duas comorbidades associadas e falha em manter o peso através de tratamentos convencionais, por um período específico de tempo [11]. O procedimento cirúrgico pode ser realizado por meio de técnicas restritivas, disabsortivas ou mistas, e têm como objetivo diminuir a quantidade de alimentos ingeridos e/ou absorvidos pelo trato digestório [12]. No entanto, apesar da intervenção cirúrgica ter pro- porcionado resultados positivos quanto à redução do peso e de comorbidades que acompanham a doença, tem-se observado deficiências nutricionais [3]. É achado comum na literatura que indivíduos submetidos à cirurgia bariátrica apresentam dieta quantitativa e qualitativamente inadequada para suprir as necessidades nutricionais [13].

Segundo Reis et al. [14], as principais carências nutricionais observadas nos indivíduos submetidos à cirurgia bariátrica são de vitaminas e minerais, principalmente vitamina B12, tiamina, ácido fólico e ferro. Desnutrição proteico calórica também pode ocorrer [14]. Assim, o sucesso da cirurgia não deve ser medido apenas pela perda do peso, mas também, pela qualidade alimentar no pós operatório e, portanto, torna-se indispensável a orientação nutricional nesse período, para assegurar a manutenção de parâmetros adequados [7,14].

Diante do exposto, o objetivo deste trabalho foi avaliar o impacto da cirurgia bariátrica no estado nutricional antropométrico e nas comorbidades associadas à SM e avaliar a adequação no consumo de micronutrientes após este procedimento cirúrgico, em mulheres de uma cidade do sudoeste de Minas Gerais.

\section{MÉTODOS}

O estudo foi do tipo observacional, transversal. Foram considerados como critérios de inclusão mulheres com idade superior a 18 anos, submetidas à cirurgia bariátrica tipo derivação gástrica em Y de Roux. A amostragem ocorreu por conveniência e as pacientes foram identificadas em consultório particular de atendimento nutricional, em Perdões, na região sul de Minas Gerais, durante o período de abril a outubro de 2010. Realizou-se contato com as pacientes, convidando-as a participar do estudo e explicando os procedimentos do mesmo. Após a confirmação de participação, as voluntárias assinaram um termo de consentimento livre e esclarecido. A pesquisa foi aprovada pelo Comitê de Ética em Pesquisa com Seres Humanos da Universidade Federal de Alfenas, sob o protocolo n ${ }^{\circ}$ 114/2010.

Foi aplicado um questionário estruturado, padronizado e pré-codificado com perguntas sobre as seguintes variáveis: idade, grau de escolaridade, renda, tempo de cirurgia e presença de comorbidades (hipertensão arterial, diabetes mellitus, dislipidemia, doenças cardiovasculares e outras doenças) antes e após a cirurgia.

Os dados sobre a antropometria antes do procedimento cirúrgico foram autorrelatados. Após a cirurgia, as variáveis antropométricas foram aferidas pelas pesquisadoras, em corte transversal. Para aferição do peso foi utilizada uma balança digital portátil 
Techine ${ }^{\circledR}$ Mod. BAL-180 BR (São Paulo - Brasil), com graduação de $100 \mathrm{~g}$ e capacidade de $180 \mathrm{~kg}$. Para aferição da estatura foi utilizado estadiômetro de madeira Altura Exata ${ }^{\circledR}$, (Belo Horizonte-Brasil) com altura máxima de $213 \mathrm{~cm}$. O IMC foi calculado pela fórmula peso em $\mathrm{kg}$ /estatura em $\mathrm{m}^{2}$ e classificado da seguinte forma: $<18,5 \mathrm{~kg} / \mathrm{m}^{2}$ baixo peso, $18,5-24,9 \mathrm{~kg} / \mathrm{m}^{2}$ eutrofia, $25-29,9 \mathrm{~kg} / \mathrm{m}^{2}$ sobrepeso, $30-34,9 \mathrm{~kg} / \mathrm{m}^{2}$ obesidade grau I, 35-39,9 kg/m² obesidade Grau II, $\geq 40 \mathrm{~kg} / \mathrm{m}^{2}$ obesidade Grau III ou mórbida, segundo a Organização Mundial da Saúde $[15,16]$. A medida da circunferência da cintura (CC) foi aferida com auxílio de uma fita métrica de material não elástico com capacidade de até $200 \mathrm{~cm}$ e precisão de $1 \mathrm{~cm}$, no ponto médio entre a décima costela e a crista ilíaca. Os pontos de corte utilizados para avaliar o risco de complicações metabólicas foram $>80 \mathrm{~cm}$ risco aumentado e $>88 \mathrm{~cm}$ risco significativamente aumentado [17]. Para análise do impacto da cirurgia bariátrica sobre as variáveis, as mulheres foram estratificadas considerando o tempo transcorrido após a cirurgia, em grupo 1 (G1 - tempo menor ou igual a 36 meses) e grupo 2 (G2 - tempo maior que 36 meses).

A avaliação dietética foi feita por meio do registro alimentar aplicado em triplicata: dois dias típicos (segunda-feira a sexta-feira) e um dia atípico (sábado, domingo ou feriado). As participantes foram orientadas a registrar em medidas caseiras todos os alimentos consumidos em cada dia, de forma detalhada em relação ao consumo de sal, açúcar e molhos de salada. Avaliou-se a composição do consumo alimentar em relação a micronutrientes (ferro, cálcio, fósforo, vitaminas hidrossolúveis e lipossolúveis e zinco) por meio do programa Dietpro versão 5i. Para análise da adequação do consumo de micronutrientes foi calculada a probabilidade de adequação usando como referências os padrões estabelecidos pelo Institute of Medicine (IOM), conhecidos como Dietary Reference Intakes (DRIs) 2002 [18].

Para construção e processamento do banco de dados utilizou-se dupla entrada e validação pelo software
EpiData (versão 3.2). As análises estatísticas foram realizadas com o software SPSS versão 17.0. O efeito da cirurgia bariátrica sobre peso e IMC foi medido pelo teste T pareado, com nível de significância de 5\%.

\section{RESULTADOS}

Foram estudadas 14 mulheres, com média de idade de $34,6 \pm 14$ anos, com tempo de realização de cirurgia compreendido entre 11 a 84 meses, sendo oito pertencentes ao G1 e seis ao G2. Os dados sociodemográficos estão relacionados na Tabela 1.

$\mathrm{Na}$ avaliação do estado nutricional antropométrico, verificou-se que nas mulheres do G1 (tempo de cirurgia menor ou igual a 36 meses) a redução média de peso corporal em relação ao referido antes da cirurgia foi de $47,83 \mathrm{~kg}$ (39,5\% de perda), enquanto naquelas do G2 (tempo de cirurgia maior que 36 meses) a perda média de peso foi de $44,5 \mathrm{~kg}(35,08 \%$ de perda). As médias de peso e de IMC antes e após a cirurgia estão descritas na Tabela 2.

Tabela 1. Variáveis referentes a 14 mulheres submetidas à cirurgia bariátrica em Perdões, região sul de Minas Gerais, durante o período de abril a outubro de 2010.

\begin{tabular}{lc}
\hline \multicolumn{1}{|c}{ Parâmetros } & $\begin{array}{c}\text { Frequência } \\
\mathbf{n}(\%)\end{array}$ \\
\hline $\begin{array}{l}\text { Renda } \\
\text { Até 3 salários mínimos }\end{array}$ & $8(57,14)$ \\
$>3$ salários Mínimos & $6(42,86)$ \\
Escolaridade & \\
$\quad$ Ensino fundamental & $3(21,43)$ \\
$\quad$ Ensino médio & $6(42,86)$ \\
$\quad$ Ensino superior & $5(35,71)$ \\
Tempo após a cirurgia & \\
$\quad$ 36 meses & $8(57,1)$ \\
$\quad$ >36 meses & $6(42,9)$ \\
Classificação do estado nutricional conforme o índice & \\
de massa corporal antes da cirurgia & \\
$\quad$ Obesidade grau I & 0 \\
$\quad$ Obesidade grau II & $3(21,43)$ \\
$\quad$ Obesidade grau III & $11(78,57)$ \\
Risco para doenças cardiovasculares conforme a medida & \\
da circunferência da cintura antes da cirurgia & \\
$\quad$ Com risco & $11(78,57)$ \\
$\quad$ Sem Risco & $3(21,43)$ \\
\hline
\end{tabular}

Tabela 2. Médias de peso e de índice de massa corporal, antes e após cirurgia bariátrica em 14 mulheres. Perdões, região sul de Minas Gerais, durante o período de abril a outubro de 2010.

\begin{tabular}{|c|c|c|c|c|c|c|}
\hline \multirow{3}{*}{ Parâmetros } & \multicolumn{6}{|c|}{ Tempo transcorrido após a cirurgia } \\
\hline & \multicolumn{3}{|c|}{$\begin{array}{c}0-36 \text { meses } \\
(n=8)\end{array}$} & \multicolumn{3}{|c|}{$\begin{array}{c}>\text { 36meses } \\
(n=6)\end{array}$} \\
\hline & Antes & Depois & $\mathbf{p}^{*}$ & Antes & Depois & $\mathbf{p}^{*}$ \\
\hline Peso (kg) & $123,07 \pm 17,27$ & $73,91 \pm 10,63$ & $<0,001$ & $124,1 \pm 14,17$ & $75,06 \pm 11,37$ & $<0,001$ \\
\hline $\mathrm{IMC}\left(\mathrm{kg} / \mathrm{m}^{2}\right)$ & $46,07 \pm 6,57$ & $27,73 \pm 4,55$ & $<0,001$ & $45,21 \pm 4,95$ & $27,33 \pm 3,72$ & $<0,001$ \\
\hline
\end{tabular}

IMC, índice de massa corporal. * Teste t pareado ao nível de 5\% de significância. 
$\mathrm{Na}$ avaliação antropométrica após a cirurgia, verificou-se que entre as oito pacientes com tempo de cirurgia igual ou inferior a 36 meses, duas $(25,0 \%)$ foram consideradas obesas, enquanto que entre as seis pacientes com tempo de cirurgia superior a 36 meses, duas $(33,3 \%)$ foram consideradas obesas (Tabela 3). Houve melhora do quadro das comorbidades associadas à obesidade, comparando as relatadas antes com as relatadas após a cirurgia (Tabela 4).
Neste estudo não se observou aversão ao consumo de alimentos fontes de cálcio e a maioria das pacientes relataram consumi-los diariamente, mas não em quantidade adequada para atingir as recomendações diárias de cálcio segundo as DRIs 2002. Em relação ao consumo de vitaminas e outros minerais, a probabilidade de adequação usando como referências as DRIs 2002 também foi em geral baixa (Tabela 5).

Tabela 3. Índice de Massa Corporal antes e após a cirurgia bariátrica, em 14 mulheres, divididas conforme o tempo transcorrido após a cirurgia. Perdões, região sul de Minas Gerais, durante o período de abril a outubro de 2010.

\begin{tabular}{|c|c|c|c|c|}
\hline \multirow{2}{*}{$\begin{array}{l}\text { Classificação do estado } \\
\text { antropométrico conforme o índice de } \\
\text { massa corporal }\end{array}$} & \multicolumn{2}{|c|}{$\begin{array}{c}\text { Tempo após a cirurgia } \\
\leq 36 \text { meses } \\
(n=8)\end{array}$} & \multicolumn{2}{|c|}{$\begin{array}{c}\text { Tempo após a cirurgia } \\
>36 \text { meses } \\
(n=6)\end{array}$} \\
\hline & $\begin{array}{l}\text { Antes } \\
\text { n (\%) }\end{array}$ & $\begin{array}{l}\text { Após } \\
\text { n (\%) }\end{array}$ & $\begin{array}{l}\text { Antes } \\
\text { n (\%) }\end{array}$ & $\begin{array}{l}\text { Após } \\
\text { n (\%) }\end{array}$ \\
\hline Obesidade III & $7(87,5 \%)$ & 0 & $5(83,33 \%)$ & 0 \\
\hline Obesidade II & $1(12,5 \%)$ & $1(12,5 \%)$ & $1(16,67 \%)$ & 0 \\
\hline Obesidade I & 0 & $1(12,5 \%)$ & 0 & $2(33,33 \%)$ \\
\hline Sobrepeso & 0 & $4(50 \%)$ & 0 & $2(33,33 \%)$ \\
\hline Eutrofia & 0 & $2(25 \%)$ & 0 & $2(33,33 \%)$ \\
\hline
\end{tabular}

Tabela 4. Prevalência de comorbidades antes e após a cirurgia bariátrica em 14 mulheres, na cidade de Perdões, região sul de Minas Gerais, durante o período de abril a outubro de 2010.

\begin{tabular}{|c|c|c|c|c|}
\hline \multirow[t]{2}{*}{ Comorbidades } & \multicolumn{2}{|c|}{$\begin{array}{c}\text { Tempo de cirurgia } \\
\leq 36 \text { meses } \\
(n=8)\end{array}$} & \multicolumn{2}{|c|}{$\begin{array}{c}\text { Tempo de cirurgia } \\
>36 \text { meses } \\
(n=6)\end{array}$} \\
\hline & $\begin{array}{l}\text { Antes } \\
\text { n (\%) }\end{array}$ & $\begin{array}{l}\text { Após } \\
\text { n (\%) }\end{array}$ & $\begin{array}{l}\text { Antes } \\
\text { n (\%) }\end{array}$ & $\begin{array}{l}\text { Após } \\
\text { n (\%) }\end{array}$ \\
\hline Hipertensão & $2(25 \%)$ & 0 & $4(66,66 \%)$ & 0 \\
\hline Doença cardiovascular & 0 & 0 & $1(16,66 \%)$ & $1(16,66 \%)$ \\
\hline Diabetes & 0 & 0 & $2(33,33 \%)$ & $1(16,66 \%)$ \\
\hline Dislipidemias & $1(12,5 \%)$ & 0 & $2(33,33 \%)$ & 0 \\
\hline
\end{tabular}

Tabela 5. Número de pacientes por cada nível de probabilidade de adequação da ingestão de micronutrientes com base nas recomendações do Institute of Medicine (Dietary Reference Intakes 2002). Avaliação de 14 mulheres submetidas à cirurgia bariátrica entre 11 a 84 meses atrás, na cidade de Perdões, região sul de Minas Gerais.

\begin{tabular}{lccccccc}
\hline \multirow{2}{*}{ Nutrientes } & \multicolumn{7}{c}{ Probabilidade de adequação } \\
\cline { 2 - 8 } & $<\mathbf{1 0} \%$ & $\mathbf{1 5 \%}$ & $\mathbf{3 0 \%}$ & $\mathbf{5 0} \%$ & $\mathbf{7 0} \%$ & $\mathbf{8 5 \%}$ & $>\mathbf{9 0} \%$ \\
\hline Ácido Ascórbico & 0 & 2 & 1 & 7 & 1 & 2 & 1 \\
Cálcio & 8 & 5 & 0 & 1 & 0 & 0 & 0 \\
Cianocobalamina & 0 & 0 & 12 & 2 & 0 & 0 & 0 \\
Ferro & 4 & 6 & 2 & 1 & 1 & 0 & 0 \\
Fósforo & 0 & 2 & 4 & 3 & 4 & 0 & 1 \\
Niacina & 0 & 1 & 1 & 10 & 2 & 0 & 0 \\
Piridoxina & 0 & 4 & 3 & 4 & 3 & 0 & 0 \\
Retinol & 0 & 0 & 5 & 4 & 5 & 0 & 0 \\
Riboflavina & 0 & 1 & 4 & 5 & 1 & 0 & 3 \\
Tiamina & 1 & 1 & 4 & 3 & 4 & 0 & 1 \\
Zinco & 0 & 0 & 8 & 6 & 0 & 0 & 0 \\
\hline
\end{tabular}

\section{DISCUSSÃO}

$\mathrm{O}$ presente estudo avaliou o estado nutricional antropométrico, comorbidades associadas à SM e adequação do consumo de micronutrientes de mulheres submetidas à cirurgia bariátrica. Observou-se que após a cirurgia ocorreu redução de peso e do IMC em todas as participantes e, na maioria dos casos, mudanças na classificação do IMC, resultados esperados após a aplicação dessa técnica cirúrgica que consiste na redução do tamanho do estômago, com redução da área absortiva. Dados da literatura descrevem que os primeiros seis meses após a cirurgia são os de perda de peso mais significativa, estabilizando-se após dois anos e podendo inclusive ocorrer recidiva do peso após certo tempo [19-23]. Essas informações são coerentes com os achados do presente estudo, que mostram uma perda ponderal maior nas mulheres com período de até 
36 meses após a cirurgia, do que naquelas com tempo superior a esse período.

Este estudo também corrobora com dados da literatura que dizem respeito à melhora das comorbidades associadas à SM (hipertensão, dislipidemias e diabetes mellitus), após a cirurgia bariátrica [22,24-27]. Em um estudo envolvendo 4.088 pacientes, 57\% foram diagnosticados como portadores de SM antes da cirurgia bariátrica e, em $46 \%$ desses pacientes, o problema foi resolvido após a cirurgia [28].

Em estudo retrospectivo realizado pela Clínica Mayo, nos Estados Unidos da América, 180 pacientes foram submetidos à cirurgia bariátrica e outros 157 foram incluídos em um programa não cirúrgico de redução de peso. Após um seguimento médio de 3,4 anos, todos os componentes da SM melhoraram no grupo cirúrgico e o número de pacientes com disfunções metabólicas diminuiu em $58 \%$, enquanto que no grupo não cirúrgico a prevalência de SM diminuiu apenas $10 \%$ [9].

Em uma metanálise com 6.587 pacientes, os investigadores concluíram que, 12 a 24 meses após a cirurgia, uma redução de $5 \mathrm{~kg} / \mathrm{m}^{2}$ no IMC correspondia a uma redução do diabetes mellitus tipo 2 em 33\%, da hipertensão arterial sistêmica em $27 \%$ e da hiperlipidemia em $20 \%$ [29]. Achado semelhante foi observado no presente estudo, no qual todas as pacientes mudaram sua classificação de IMC, passando de obesidade mórbida para níveis de obesidade leve, sobrepeso ou eutrofia, além de $100 \%$ de correção do quadro de hipertensão e dislipidemia, independentemente do tempo pós cirurgia e, naquelas com tempo de cirurgia superior a 36 meses, observouse redução de $50 \%$ nos casos de diabetes. Em uma paciente portadora de doença cardiovascular, não foi observada melhora.

Segundo De Lorenzo et al. [27], a perda de peso gera diminuição da resistência periférica à insulina, uma vez que os tipos celulares presentes no tecido adiposo são responsáveis pelo aumento nos níveis circulantes de citocinas pró inflamatórias, entre elas, TNF- $\alpha$, IL-6, leptina e proteína C-reativa, que exercem influência em mecanismos inflamatórios [27].

A avaliação dietética mostrou que, em média, houve inadequação na ingestão dos seguintes micronutrientes: cálcio, ferro, vitamina B12, vitamina
B6 e zinco. A deficiência desses micronutrientes pôde ser observada em diversos trabalhos, e é consequência principalmente da redução da ingestão de alimentos após a cirurgia bariátrica e da má absorção de alguns nutrientes, dependendo da técnica cirúrgica utilizada e de aversões alimentares específicas [30-35].

Embora as mulheres participantes do estudo não relatarem aversão a alimentos ricos em cálcio, observou-se que a quantidade era inadequada para suprir as necessidades mínimas desse mineral. Um estudo de revisão sistemática também encontrou adequação de cálcio menor ou igual a $50 \%$ em pacientes submetidos a cirurgia bariátrica [31]. A deficiência de cálcio pode comprometer funções tais como de mensageiro intracelular, cofator para enzimas e proteínas extracelulares e manutenção da massa óssea.

Além da inadequação na ingestão de cálcio, as ingestões de ferro, vitamina B12, zinco e vitamina B6 também foram inadequadas, pela redução no consumo de alimentos fonte desses micronutrientes [31,35]. Além da baixa ingestão, a vitamina B12 pode ter sua absorção comprometida dependendo da técnica utilizada no procedimento cirúrgico $[30,33]$.

Neste estudo, a cirurgia bariátrica mostrou-se um método eficaz no tratamento da obesidade e no controle das comorbidades associadas à SM. No entanto, a presença de carências nutricionais apontam para maior necessidade de cuidado nutricional no pós operatório em pacientes submetidos a esse procedimento cirúrgico.

O presente estudo apresenta algumas limitações. A amostra do estudo foi pequena, o que reduz a validade externa do trabalho. As comorbidades antes e após o tratamento cirúrgico e o peso anterior ao tratamento foram autorrelatados, podendo apresentar viés de informação. Os dados do consumo alimentar antes da cirurgia, para efeito de comparação, não foram obtidos.

Apesar das limitações apresentadas, o estudo permitiu verificar os efeitos da cirurgia bariátrica na promoção da saúde, com impacto positivo na redução do IMC e das comorbidades associadas à $\mathrm{SM}$, indicando ainda a necessidade de direcionar intervenções nutricionais intensivas para prevenção de carências nutricionais em indivíduos submetidos a essa modalidade de tratamento. 


\section{NOTA}

\section{Declaração de conflitos de interesse}

As autoras declaram não haver conflitos de interesse relevantes ao conteúdo deste estudo.

\section{REFERÊNCIAS}

1. Grundy SM. Metabolic syndrome update. Trends Cardiovasc Med. 2016;26(4):364-73. http://dx.doi.org/10.1016/j.tcm.2015.10.004

2. Shuai X, Tao K, Mori M, Kanda T. Bariatric surgery for metabolic syndrome in obesity. Metab Syndr Relat Disord. 2015;13(4):149-60. http://dx.doi.org/10.1089/met.2014.0115

3. Silva PRB, Souza MR, Silva EM, Silva SA. Nutritional status and life quality in patients undergoing bariatric surgery. Arq Bras Cir Dig. 2014;27 (1): 35-8. http://dx.doi.org/10.1590/S0102-6720201400S100009

4. World Health Organization. Obesity and Overweight [Internet]. Genebra; 2015 [updated 2015 Jan; cited 2016 Mar 14]. Available from http://www.who.int/mediacentre/factsheets/fs311/en/

5. Portal Brasil. Ministério da Saúde. Mais da metade dos adultos está acima do peso [Internet]. Brasília; 2015 [updated 2015 Aug; cited 2016 Mar 14]. Available from: http://www.brasil.gov.br/saude/2015/08/mais-da-metade-dos-adultos-estao-acima-do-peso

6. Arble DM, Sandoval DA, Seeley RJ. Mechanisms underlying weight loss and metabolic improvements in rodent models of bariatric surgery. Diabetologia. 2015;58(2):211-20. http://dx.doi.org/10.1007/s00125-014-3433-3

7. Martini F, Anty R, Schneck AS, Casanova V, Iannelli A, Gugenheim J. Predictors of metabolic syndrome persistence 1 year afte laparoscopic Roux-en-Y gastric bypass. Surg Obes Relat Dis. 2015;11(5):1054-60. http://dx.doi.org/10.1016/j.soard.2015.02.019

8. Ikramuddin S, Buchwald H. How bariatric and metabolic operations control metabolic syndrome. Br J Surg. 2011;98 (10):1339-41. http:// dx.doi.org/10.1002/bjs.7652

9. Batsis JA, Romero-Corral A, Collazo-Clavell ML, Sarr MG, Somers VK, Lopez-Jimenez F. Effect of bariatric surgery on the metabolic syndrome: a population-based, long-term controlled study. Mayo Clin Proc. 2008;83(8):897-907. http://dx.doi.org/10.1016/S00256196(11)60766-0

10. Faria G, Pestana D, Aral M, Preto J, Norberto S, Calhau C, Guimarães JT, Taveira-Gomes A. Metabolic score: Insights on the development and prediction of remission of metabolic syndrome after gastric bypass. Ann Surg. 2014;260 (2):279-86. http://dx.doi.org/10.1097/ SLA.0000000000000686

11. American Society for Metabolic and Bariatric Surgery. Who is Candidate for Bariatric Surgery? [Internet]. Gainesville; 2016 [cited 2016 ; Mar 14]. Available fro https://asmbs.org/patients/who-is-a-candidate-for-bariatric-surgery

12. American Society for Metabolic and Bariatric Surgery. Bariatric Surgery Procedures [Internet]. Gainesville; 2016 [cited 2016 ; Mar 14]. Available from: https://asmbs.org/patients/bariatric-surgery-procedures

13. Bastos ECL, Barbosa EMWG, Soriano GMS, Santos EA, Vasconcelos SML. Fatores determinantes do reganho ponderal no pós-operatório de cirurgia bariátrica. Arq Bras Cir Dig. 2013;26(suppl.1):26-32. http://dx.doi.org/10.1590/S0102-67202013000600007

14. Reis TN, Da Silva MMF, Silveira VFSB, Andrade RD. Resultados da cirurgia bariátrica a longo prazo. Ciênc Praxis. 2012;6(10):43-52.

15. Castro SH, Mato HJ, Gomes MB. Parâmetros antropométricos e síndrome metabólica em diabetes tipo 2. Arq Bras Endocrinol Metab. 2006;50(3):450-5. http://dx.doi.org/10.1590/S0004-27302006000300007

16. World Health Organization. Physical Status: The Use and Interpretation of Anthropometry. WHO Technical Report Series 854. Geneva: WHO; 1995.

17. Associação Brasileira para o Estudo da Obesidade e da Síndrome Metabólica. Diretrizes Brasileiras de Obesidade. 3a ed. Itapevi, SP: AC Farmacêutica; 2009.

18. American Dietetic Association. Practice paper of the American Dietetic Association: Using the Dietary Reference Intakes. J Am Diet Assoc. 2011;111:762-70. http://dx.doi.org/10.1016/j.jada.2011.03.022

19. Costa LD, Valezi AC, Matsuo T, Dichi I, Dichi JB. Repercussão da perda de peso sobre parâmetros nutricionais e metabólicos de pacientes obesos graves após um ano de gastroplastia em Y-de-Roux. Rev Col Bras Cir. 2010;37(2):96-101. http://dx.doi.org/10.1590/S010069912010000200005

20. Vicente MA, Freitas AR. Avaliação das alterações nutricionais, clínicas e bioquímicas em pacientes submetidos à cirurgia bariátrica nos períodos pré e pós-cirúrgico. RBONE. 2011;5(28):333-43.

21. Boscatto EC, Duarte MFS, Gomes MA. Comportamentos ativos e percepção da saúde em obesos submetidos à cirurgia bariátrica. Rev Bras Ativ Fís Saúde. 2011;16(1):43-7.

22. Svane MS, Madsbad S. Bariatric surgery - effects on obesity and related co-morbidities. Curr Diabetes Rev. 2014 May;10(3):208-14. http://dx.doi.org/10.2174/1573399810666140616144141

23. Sjöström L, Lindroos AK, Peltonen M, Torgerson J, Bouchard C, Carlsson B, Dahlgren S, Larsson B, Narbro K, Sjöström CD, Sullivan M, Wedel H; Swedish Obese Subjects Study Scientific Group. Lifestyle, diabetes, and cardiovascular risk factors 10 years after bariatric surgery. N Engl J Med. 2004 Dec 23;351(26):2683-93. http://dx.doi.org/10.1056/NEJMoa035622

24. Silva-Neto EF, Vázquez, CMP, Soares FM, Silva DG, Souza MFC, Barbosa KBF. Cirurgia bariátrica reverte risco metabólico em pacientes assistidos em nível ambulatorial. Arq Bras Cir Dig. 2014;27(1):38-42. http://dx.doi.org/10.1590/S0102-67202014000100010

25. Hess S, Tramontini J, Canuto R. Fatores associados à síndrome metabólica em adultos atendidos em um ambulatório de nutrição. Sci Med. 2014;24(1):33-8. http://dx.doi.org/10.15448/1980-6108.2014.1.14815 
26. Dixon JB, Zimmet P, Alberti KG, Rubino F; International Diabetes Federation Taskforce on Epidemiology and Prevention. Bariatric surgery: an IDF statement for obese Type 2 diabetes. Arq Bras Endocrinol Metab. 2011;55(6):367-82. http://dx.doi.org/10.1590/S000427302011000600003

27. De Lorenzo, Soldati L, Sarlo F, Calvani M, Di Lorenzo N, Di Renzo L. New obesity classification criteria as a tool for bariatricsurgery indication. World J Gastroenterol. 2016 Jan 14;22(2):681-703. http://dx.doi.org/10.3748/wjg.v22.i2.681

28. Coleman KJ, Huang YC, Koebnick C, Reynolds K, Xiang AH, Black MH, Alskaf S. Metabolic syndrome is less likely to resolve in Hispanics and non- Hispanic blacks after bariatric surgery. Ann Surg. 2014;259 (2):279-85. http://dx.doi.org/10.1097/SLA.0000000000000258

29. Ricci C, Gaeta M, Rausa E, Macchitella Y, Bonavina L. Early impact of bariatric surgery on type II diabetes, hypertension, and hyperlipidemia: A systematic review, meta-analysis and meta-regression on 6,587 patients. Obes Surg. 2014;24(4):522-8. http://dx.doi. org/10.1007/s11695-013-1121-X

30. Santos CF, Amado T, Sandri YP, Frizzo MN. Alterações dos parâmetros laboratoriais em paciente submetidos à cirurgia bariátrica. Rev Saúde Inst Cenecista. 2014;7(13):13-4.

31. Leiro LS, Melendez-Araújo MS. Adequação de micronutrientes da dieta de pacientes submetidos à cirurgia bariátrica. Comun Ciênc Saúde. 2013;24(4):353-62.

32. Bordalo LA, Salesteixeira TF, Bressan J, Mourão DM. Cirurgia bariátrica: como e por que suplementar. Rev Assoc Med Bras. 2011;57(1): 113-20. http://dx.doi.org/10.1590/S0104-42302011000100025

33. Bordalo LA, Mourão DM, Bressan J. Deficiências nutricionais após cirurgia bariátrica: porque ocorrem? Acta Med Port. 2011;24(S4): 1021-8.

34. British obesity and metabolic surgery society. Guidelines on perioperative and postoperative biochemical monitoring and micronutrient replacement for patients undergoing bariatric surgery [Internet] 2014. [updated 2016 Sep; cited 2016 Mar 14]. Available from: http://www. bomss.org.uk/wp-content/uploads/2014/09/BOMSS-guidelines-Final-version1Oct14.pdf

35. Aron-Wisnewsky J, Verger EO, Bounaix C, Dao MC, Oppert JM, Bouillot JL, Chevallier JM, Clément K. Nutritional and Protein Deficiencies in the Short Term following Both Gastric Bypass and Gastric Banding. PLoS ONE. 2016;11(2):e0149588. http://dx.doi. org/10.1371/journal.pone.0149588 